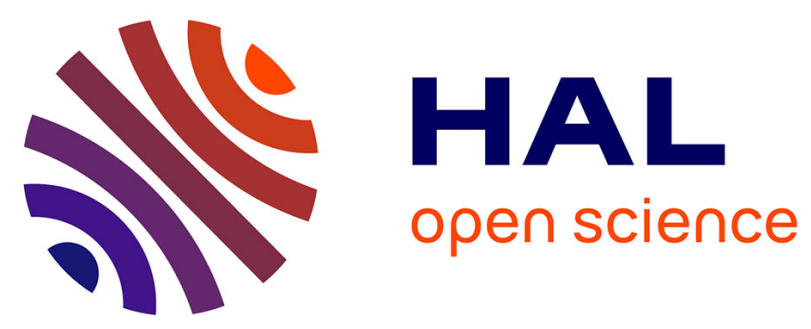

\title{
Continuous and discrete-time stability of a robust set-valued nested controller
}

\author{
Felix Miranda-Villatoro, Fernando Castaños, Bernard Brogliato
}

\section{To cite this version:}

Felix Miranda-Villatoro, Fernando Castaños, Bernard Brogliato. Continuous and discretetime stability of a robust set-valued nested controller. Automatica, 2019, 107, pp.406-417. 10.1016/j.automatica.2019.06.003 . hal-01944920v2

\section{HAL Id: hal-01944920 \\ https://inria.hal.science/hal-01944920v2}

Submitted on 26 Jun 2019

HAL is a multi-disciplinary open access archive for the deposit and dissemination of scientific research documents, whether they are published or not. The documents may come from teaching and research institutions in France or abroad, or from public or private research centers.
L'archive ouverte pluridisciplinaire $\mathbf{H A L}$, est destinée au dépôt et à la diffusion de documents scientifiques de niveau recherche, publiés ou non, émanant des établissements d'enseignement et de recherche français ou étrangers, des laboratoires publics ou privés. 


\title{
Continuous and discrete-time stability of a robust set-valued nested controller
}

\author{
Félix A. Miranda-Villatoro ${ }^{a}$, Fernando Castaños $^{b}$, Bernard Brogliato $^{c}$ \\ ${ }^{a}$ University of Cambridge, Department of Engineering, Trumpington Street, Cambridge CB2 1PZ \\ ${ }^{\mathrm{b}}$ Departamento de Control Automático, Cinvestav-IPN, 2508 Av. Instituto Politécnico Nacional, 07360, Mexico City, Mexico \\ ${ }^{\mathrm{c}}$ Univ. Grenoble-Alpes, Inria, CNRS, Grenoble INP, LJK, 38000 Grenoble, France
}

\begin{abstract}
We propose a set-valued controller with a signum multifunction nested inside another one. We prove that the controller is well posed and achieves robust ultimate boundedness in the presence of mismatched, non-vanishing disturbances. Even more, the selected output can be made arbitrarily small. Also, by applying an implicit/explicit Euler scheme similar to the one introduced by Acary and Brogliato [15] for matched disturbances, we derive a selection strategy for the discrete-time implementation of the set-valued control law. Simulations demonstrate that the discrete scheme diminishes chattering substantially, compared with a fully explicit method.
\end{abstract}

Key words: Multivalued mapping; Sliding-mode control; Discrete-time systems; Robust control; Disturbance rejection; Lyapunov methods; Backstepping controller.

\section{Introduction}

Since its appearance, sliding-mode control has been studied in detail because of its robustness against matched disturbances and parametric uncertainty [14]. However, the controllers of this type are known to be fragile in the presence of mismatched disturbances, that is, disturbances that affect the system through a channel not shared by the control input, and which in consequence, cannot be eliminated by applying the conventional methods developed in [1].

Several solutions have been proposed in order to overcome the problem of high sensitivity to mismatched disturbances. The combination of conventional (first-order) sliding-mode control with other robust techniques such as $\mathcal{H}_{\infty}$ is one of such solutions [5]. Others [6-8], are based on the use of high-order sliding-mode control techniques. In $[6,8]$, the main idea is to estimate the disturbances first and then apply a control law that compensates them while fulfilling the control objective (regulation or tracking of the desired output in finite time). Another approach makes use of the so-called robust control Lyapunov functions [9]. The authors prove that the

Email address: castanos@ieee.org (Fernando Castaños). knowledge of such a Lyapunov function yields a control law that is also optimal in some meaningful sense. A nonsmooth version of the technique is developed in [10].

Mismatched uncertainty may appear in the form of parametric uncertainty or unmodeled dynamics. Then, in some cases, the solution to a set of linear matrix inequalities can be used to design a sliding surface such that the sliding motions are completely invariant with respect to the mismatched uncertainty $[11,12]$. This approach requires, however, the mismatched uncertainty to vanish at the desired equilibrium.

A distinctive feature of first-order sliding-mode control is the finite-time convergence of the state towards the so-called sliding surface. However, if the sliding surface is linear, the state will approach the origin only exponentially fast, even while in the sliding regime. To achieve finite-time convergence to the origin, terminal sliding-mode control was proposed $[13,14]$. For a system of the form (10) (see below) without mismatched perturbations, i.e., when $\zeta_{1}\left(t, x_{1}\right) \equiv 0$, one defines a sliding variable $s(x)=x_{2}+\left|x_{1}\right|^{p} \operatorname{sgn} x_{1}$ with $0<p<1$. It is not difficult to verify that, along the sliding surface $\left\{x \in \mathbb{R}^{2} \mid s(x)=0\right\}$, the state converges indeed to the origin in finite time. In this paper, we study the limiting case $p=0$, which results in a controller with 
a signum function nested inside another one. In other words, not only the control action is discontinuous, but the switching surface is discontinuous as well. The controlled system is studied under a differential-inclusions perspective.

A discrete-time version of the controller is developed using an implicit/explicit Euler scheme. The idea follows along the lines of $[15-21,12]$, in which a backward Euler scheme results in a control law implicitly defined by an algebraic inclusion (i.e., a generalized equation). Intuitively, the algebraic inclusion may be thought of as the discrete counterpart of Filippov's differential inclusion. We say that a control value is adequately selected if it is a solution of such inclusion, in which case the control law is able to compensate the matched disturbances while alleviating the digital chattering.

Reference [22] is closely related to our work. The authors also consider the case $p=0$, but then use a sigmoidal approximation in place of the true signum multifunction. In this regard, our work can also be understood as a limiting case of the controller proposed in [22].

In this context, it is worth mentioning the nested sliding mode controllers presented in [23], which have signum functions inside other signum functions as well. This family of controllers is capable of steering the complete state to the origin in finite time by establishing a higherorder sliding-motion. However, neither mismatched perturbations nor time-discretization are analyzed.

Also related to our work is [24], where the problem of control with amplitude- and rate-saturation constraints is addressed with a nested sigmoidal controller.

\section{Contributions}

We present results in continuous and discrete time. For continuous time, the main result is the ultimate boundedness of the closed-loop system in the presence of mismatched disturbances. We first prove the wellposedness (existence of solutions) of the closed-loop system with the nested algorithm, by using a set-valued framework. Closed-loop trajectories can be decomposed in three phases: a reaching phase, a sliding motion along a continuous segment of the switching surface and an oscillatory phase around the corners of the switching surface. Practical stability is shown by means of a single Lyapunov function that is suitable for the three phases. The function is Lipschitz continuous but nondifferentiable, which requires the application of the set-valued derivative defined in [25]. Unlike many results on ultimate boundedness, the analysis yields an explicit relation between the ultimate bounds on the trajectories and the controller parameters.

The main challenge in a discretization scheme like the one outlined above is guaranteeing the solvability of the resulting inclusion. This has been typically achieved by exploiting the maximal monotone nature of the signum multifunction. Unfortunately, the composition of two signum multifunctions is no longer maximal monotone. To overcome this problem, the selection process is split into two parts, yielding a discrete-time scheme reminiscent of backstepping. The resulting control law is capable of rejecting both matched and unmatched disturbances with practically no chattering.

\section{Paper structure}

The paper is organized as follows: Section 2 recalls some results on stability theory in the nonsmooth setting. Section 4 is dedicated to well-posedness and stability issues of the closed-loop system in continuous time, whereas Section 5 studies the discrete-time counterpart of the nested controller. We present numerical results and the comparison of the implicit discretization against explicit techniques. Finally, the paper ends with conclusions and possible future work.

\section{Preliminaries}

Throughout this paper we deal with set-valued maps, that is, maps that evaluate to a subset of the range at each point of their domain. A set-valued map with domain $\mathbb{R}^{n}$ and taking values on the power set of $\mathbb{R}^{m}$ is denoted as $\mathbf{F}: \mathbb{R}^{n} \rightrightarrows \mathbb{R}^{m}$. For example, the set-valued function $\operatorname{Sgn}: \mathbb{R} \rightrightarrows[-1,1] \subset \mathbb{R}$ is given by

$$
\operatorname{Sgn}(x):=\left\{\begin{array}{ll}
\operatorname{sgn}(x) & \text { if } x \neq 0 \\
{[-1,1]} & \text { otherwise }
\end{array},\right.
$$

whereas the single-valued function $\operatorname{sgn}: \mathbb{R} \backslash\{0\} \rightarrow \mathbb{R}$ is defined as

$$
\operatorname{sgn}(x):=\left\{\begin{array}{ll}
-1 & \text { if } x<0 \\
1 & \text { if } x>0
\end{array} .\right.
$$

Note that the single-valued signum function is undefined at $x=0$.

Definition 1 A set-valued map $\mathbf{F}: X \rightrightarrows Y$ is called upper semi-continuous (usc) at $x_{0} \in X$ if, for any open neighborhood $M$ of $\mathbf{F}\left(x_{0}\right)$, there exists a neighborhood $N$ of $x_{0}$ such that $\mathbf{F}(N) \subset M$. The set-valued map $\mathbf{F}$ is upper semi-continuous if it is so at every $x_{0} \in X$.

The graph of a given map $\mathbf{F}$ is defined as

$$
\operatorname{Graph} \mathbf{F}=\left\{(x, y) \in \mathbb{R}^{n} \times \mathbb{R}^{m} \mid y \in \mathbf{F}(x)\right\} .
$$

Let $\mathbf{F}$ and $\mathbf{G}$ be two set-valued maps, the composition map $\mathbf{F} \circ \mathbf{G}$ is given by the formula

$$
\mathbf{F} \circ \mathbf{G}(x):=\cup_{y \in \mathbf{G}(x)} \mathbf{F}(y) .
$$


Proposition $2([\mathbf{2 6}, \mathbf{p . 4 1}])$ Let $\mathbf{F}$ and $\mathbf{G}$ be two setvalued usc maps. Then, the composition map $\mathbf{F} \circ \mathbf{G}$ is also usc.

Set-valued maps can be used, e.g., to define generalized equations of the form $0 \in F(x)$, where the goal is to find the values of $x$ for which the inclusion is satisfied.

Besides usual set operations such as union and intersection, we consider addition and multiplication. Let $\mathcal{C}_{1} \subset \mathbb{R}^{n}$ and $\mathcal{C}_{2} \subset \mathbb{R}^{n}$ be two sets. The sum is the set

$$
\mathcal{C}_{1}+\mathcal{C}_{2}=\left\{\sigma \in \mathbb{R}^{n} \mid \sigma=\sigma_{1}+\sigma_{2}, \sigma_{1} \in \mathcal{C}_{1}, \sigma_{2} \in \mathcal{C}_{2}\right\} .
$$

For $n=1$ we define the product

$$
\mathcal{C}_{1} \cdot \mathcal{C}_{2}=\left\{\sigma \in \mathbb{R} \mid \sigma=\sigma_{1} \cdot \sigma_{2}, \sigma_{1} \in \mathcal{C}_{1}, \sigma_{2} \in \mathcal{C}_{2}\right\} .
$$

The sum and the product between an element and a set is defined in the obvious way.

We denote the open unit ball as

$$
\mathcal{B}_{n}=\left\{x \in \mathbb{R}^{n} \mid\|x\|<1\right\} .
$$

Given a set $\mathcal{C}$, the closure and the interior are denoted by $\operatorname{cl}(\mathcal{C})$ and $\operatorname{int}(\mathcal{C})$, respectively. The boundary $\operatorname{cl}(\mathcal{C}) \backslash \operatorname{int}(\mathcal{C})$ is denoted by bd $(\mathcal{C})$. If $\mathcal{C} \subset \mathbb{R}^{n}$ and $x \in \mathbb{R}^{n}$, the distance between them is defined as

$$
|x|_{\mathcal{C}}=\inf _{w \in \mathcal{C}}\|x-w\|,
$$

while the inner product is defined as

$$
\langle\mathcal{C}, x\rangle=\{\langle\sigma, x\rangle \mid \sigma \in \mathcal{C}\} .
$$

Whenever $\mathcal{C}$ is closed, we define the projection of $x$ onto $\mathcal{C}$ as

$$
\operatorname{Proj}(x, \mathcal{C})=\underset{w \in \mathcal{C}}{\arg \min }\|x-w\| .
$$

Consider the non-autonomous differential inclusion

$$
\dot{x} \in \mathbf{G}(t, x), \quad x(0)=x_{0} .
$$

The following theorem establishes conditions for the existence of solutions of the differential inclusion.

Theorem 3 [26, Theorem 3, p. 98] Let:

(1) $Q \subset \mathbb{R} \times \mathbb{R}^{n}$ be an open subset containing $\left(0, x_{0}\right)$.

(2) $\mathbf{G}$ be an upper semicontinuous map from $Q$ into the non-empty closed convex subsets of $\mathbb{R}^{n}$.

(3) The map $(t, x) \mapsto m(\mathbf{G}(t, x))$ be locally compact with $m(\mathbf{G}(t, x))$ the element of $\mathbf{G}(t, x)$ with the smallest norm.
Then, there exist $T>0$ and an absolutely continuous function $x(\cdot)$ defined on $[0, T]$, a solution to the differential inclusion (2).

We will make use of the notion of stability of sets and the corresponding Lyapunov theory that has been developed in the context of nonsmooth analysis and differential inclusions. In particular, we will take into account the possibility that the solutions to a given differential inclusion may not be unique. Following [27], we will say that a set $\mathcal{C}$ is stable if, for any $\varepsilon>0$, there exists a $\delta>0$ such that, for any initial condition $x_{0}$ satisfying $\left|x_{0}\right|_{\mathcal{C}}<\delta$, it follows that $|x(t)|_{\mathcal{C}}<\varepsilon$ for all time $t>0$ and all solutions passing through $x_{0}$ at $t=0$. Moreover, the set $\mathcal{C}$ is said to be globally asymptotically stable if all solutions satisfy $|x(t)|_{\mathcal{C}} \rightarrow 0$ as $t \rightarrow+\infty$.

We will temporarily restrict our attention to the autonomous differential inclusion

$$
\dot{x} \in \mathbf{F}(x), \quad x(0)=x_{0},
$$

where $\mathbf{F}: \mathbb{R}^{n} \rightrightarrows \mathbb{R}^{n}$ is an usc map. Let $V: \mathbb{R}^{n} \rightarrow \mathbb{R}$ be a possibly nondifferentiable but Lipschitz continuous function. The set-valued derivative of $V$ along the trajectories of (3) is defined as [25]

$$
\begin{aligned}
\mathcal{L}_{\mathbf{F}} V(x):=\{a \in \mathbb{R} \mid \exists v \in \mathbf{F}(x) & \text { such that }\langle\partial V(x), v\rangle=\{a\}\},
\end{aligned}
$$

where the term $\partial V(x)$ refers to Clarke's generalized gradient of the function $V$ at the point $x$. Formally, let $\Omega_{V}$ be the zero-measure set where the function $V$ is nondifferentiable and let $\Omega$ be any subset of $\mathbb{R}^{n}$ with measure zero, then $[25]$

$$
\partial V(x):=\operatorname{conv}\left\{\lim \nabla V\left(x_{i}\right) \mid x_{i} \rightarrow x, x_{i} \notin \Omega \cup \Omega_{V}\right\},
$$

where the operator conv stands for convex closure.

Remark 4 When $V$ is differentiable at $x, \partial V(x)$ is single-valued and the set-valued derivative simplifies to

$$
\mathcal{L}_{\mathbf{F}} V(x)=\langle\partial V(x), F(x)\rangle .
$$

In the following, we agree that $\max \mathcal{L}_{\mathrm{F}} V(x)=-\infty$ whenever $\mathcal{L}_{\mathbf{F}} V(x)=\emptyset$.

Definition 5 A function $f: \mathbb{R}^{n} \rightarrow \mathbb{R}$ is said to be regular at $x \in \mathbb{R}^{n}$ if, for all $z \in \mathbb{R}^{n}$, the usual one-sided derivative

$$
\lim _{\lambda \rightarrow 0^{+}} \frac{f(x+\lambda z)-f(x)}{\lambda}
$$

exists and is equal to the generalized directional derivative

$$
\limsup _{\substack{s \rightarrow x \\ \lambda \rightarrow 0^{+}}} \frac{f(s+\lambda z)-f(s)}{\lambda} .
$$


The function $f$ is called regular if it is regular at each $x \in \mathbb{R}^{n}$.

Proposition 6 ([28, Proposition 2]) Let $f$ be Lipschitz near $x$.

i) If $f$ is convex, then $f$ is regular at $x$.

ii) A finite linear combination (by nonnegative scalars) of functions regular at $x$, is regular at $x$.

To compute Clarke's generalized gradient, we will use the following nonsmooth chain rule, adapted from [29, Theorem 2.3.9]

Proposition 7 Let $f: \mathbb{R}^{n} \rightarrow \mathbb{R}$ be given as $f=g \circ h$, where $h: \mathbb{R}^{n} \rightarrow \mathbb{R}$ is a Lipschitz continuous function and $g: \mathbb{R} \rightarrow \mathbb{R}$ is continuously differentiable. Then,

$$
\partial f(x)=\nabla g(h(x)) \partial h(x) .
$$

The following is a generalization of [25, Theorem 2] to the stability of sets. Its proof resembles the one given in $[30]$.

Theorem 8 Consider the differential inclusion (3) with $F$ usc with compact and convex values. Let $\Gamma \subset \mathbb{R}^{n}$ be a compact subset of $\mathbb{R}^{n}$ and let $V: \mathbb{R}^{n} \rightarrow \mathbb{R}$ be a regular, radially unbounded and locally Lipschitz continuous function satisfying $V(x) \geq 0$ for all $x \in \mathbb{R}^{n}$ and

$$
V(x)>0 \quad \text { for all } x \in \mathbb{R}^{n} \backslash \Gamma .
$$

Consider the set $\Omega_{c}:=\left\{x \in \mathbb{R}^{n} \mid V(x) \leq c\right\}$ and define

$$
c^{*}:=\min \left\{c \mid \Gamma \subseteq \Omega_{c}\right\} .
$$

Thus, the set $\Omega_{c^{*}}$ is the smallest sublevel set of $V$ that contains $\Gamma$. Under the aforementioned assumptions, the set $\Omega_{c^{*}}$ is globally asymptotically stable if

$$
\max \mathcal{L}_{\mathbf{F}} V(x) \leq-W(x),
$$

where $W: \mathbb{R}^{n} \rightarrow \mathbb{R}$ is continuous and positive definite with respect to $\Gamma$. That is,

$$
W(x)>0 \quad \text { for all } x \notin \Gamma .
$$

PROOF. Recalling from [25, Lemma 1] that the derivative of $V$ along the trajectories of (3) exists almost everywhere and satisfies $\frac{d}{d t} V(x(t)) \in \mathcal{L}_{\mathbf{F}} V(x(t))$ almost everywhere, it follows that the sets $\Omega_{c}$ are positively invariant for all $c \geq c^{*}$. On the other hand, the fact that $V$ is radially unbounded together with the positivity assumptions imply the boundedness of the sublevel sets $\Omega_{c}$ for all $c \geq 0$. This also establishes globality.
Define the neighborhood $N_{\varepsilon}=\left\{\left.x \in \mathbb{R}^{n}|| x\right|_{\Omega_{c^{*}}}<\varepsilon\right\}$ and let

$$
\tilde{c}=\min _{x \in \operatorname{bd}\left(N_{\varepsilon}\right)} V(x) .
$$

Then, $\tilde{c}>c^{*}$. To see this, simply note that $|x|_{\Omega_{c^{*}}}=\varepsilon$ implies $x \notin \Omega_{c^{*}}$, so that $V(x)>c^{*}$. Choose any $\hat{c} \in\left(c^{*}, \tilde{c}\right)$ and observe that $\Omega_{c^{*}} \subset \Omega_{\hat{c}}$ and $\Omega_{\hat{c}} \cap N_{\varepsilon} \neq \emptyset$. Moreover, $\Omega_{\hat{c}} \subset N_{\varepsilon}$. To prove the latter, assume by contradiction that there exists a $\xi$ in $\Omega_{\hat{c}} \cap \operatorname{bd}\left(N_{\varepsilon}\right)$. We have

$$
\xi \in \operatorname{bd}\left(N_{\varepsilon}\right) \Longrightarrow V(\xi) \geq \tilde{c} \quad \text { and } \quad \xi \in \Omega_{\hat{c}} \Longrightarrow V(\xi) \leq \hat{c},
$$

so that $\hat{c} \geq \tilde{c}$, the desired contradiction.

Now, choose $\mu \in\left(0, \hat{c}-c^{*}\right)$. It follows from the continuity of $V$ that, for every $x \in \operatorname{bd}\left(\Omega_{c^{*}}\right)$, there exists a $\delta_{x}$ depending on $x$ and $\mu$ such that

$$
\|\xi-x\|<\delta_{x} \Longrightarrow|V(\xi)-V(x)|<\mu .
$$

Set $\delta=\min _{x \in \operatorname{bd}\left(\Omega_{c^{*}}\right)} \delta_{x}$ (the minimum exists by the compacity of $\Omega_{c^{*}}$ ). Let $\xi$ be any point in $N_{\delta} \backslash \Omega_{c^{*}}$ and let $x \in \operatorname{Proj}\left(\xi, \Omega_{c^{*}}\right)$. We have

$$
\begin{aligned}
V(\xi) & =V(x)+V(\xi)-V(x) \\
& \leq V(x)+|V(\xi)-V(x)| \\
& <c^{*}+\mu .
\end{aligned}
$$

Since $c^{*}+\mu<\hat{c}$, we finally obtain

$$
N_{\delta} \subset \Omega_{\hat{c}} \subset N_{\varepsilon},
$$

which implies that trajectories starting in $N_{\delta}$ cannot leave $N_{\varepsilon}$.

Asymptotic stability will be established by showing that

$$
|x(t)|_{\Omega_{c^{*}}} \rightarrow 0 \quad \text { as } t \rightarrow \infty .
$$

We will use a contradiction argument. Suppose that

$$
x(t) \notin \Omega_{c^{*}} \quad \text { for all } t \geq 0,
$$

then $V(x(t))$ is monotonically decreasing and, since it also bounded from below,

$$
V(x(t)) \rightarrow \bar{c}
$$

for some $\bar{c}>c^{*}$. Again by continuity of $V$, there exists a $b>0$ such that $N_{b} \subset \Omega_{\bar{c}}$. The limit $V(x(t)) \rightarrow \bar{c}$ implies that the trajectory $x(t)$ lies outside the neighborhood $N_{b}$. Let

$$
\gamma=\min \left\{\left.W(x)|b \leq| x\right|_{\Omega_{c^{*}}} \leq \varepsilon\right\} .
$$

By (7), we have $\gamma>0$. It follows from (6) that

$$
V(x(t)) \leq V(x(0))-\gamma t .
$$


The fact that the right-hand side will eventually become less than $c^{*}$ contradicts the assumption that $\bar{c}>c^{*}$.

Observe that no assumptions have been imposed on the nature of the trajectories $x(\cdot)$, other than they are solutions of (3). Hence, the inclusion (8) (stability) and the limit (9) (convergence) hold for all solutions of (3).

\section{Problem Statement}

Consider the system

$$
\begin{aligned}
\dot{x}_{1} & =x_{2}+\zeta_{1}\left(t, x_{1}\right) \\
\dot{x}_{2} & =u+\zeta_{2}(t, x), \\
y & =x_{1}
\end{aligned},
$$

where $x_{i} \in \mathbb{R}$ represent the states of the system, $\zeta_{i} \in \mathbb{R}$ account for external disturbances and uncertain dynamics, $i=1,2$, and $u, y \in \mathbb{R}$ are the control input and desired output, respectively.

Assumption 9 The disturbance terms $\zeta_{i}: \mathbb{R} \times \mathbb{R}^{i} \rightarrow \mathbb{R}$, $i=1,2$, are measurable with respect to the first argument, continuous with respect to the second argument and uniformly bounded by positive constants $w_{i}$. That is, $\zeta_{i} \in w_{i} \cdot \mathcal{B}_{1}$ for all $t$ and all $x$.

Objective: To regulate the output $y=x_{1}$ to a neighbourhood of the origin in the presence of disturbances $\zeta_{i}, i=1,2$.

Note that, even for this simple plant, the task of regulating the output is a challenging problem because of the presence of the unmatched disturbance $\zeta_{1}$.

A motivation for studying (10) is the following. Briefly consider a more general system

$$
\begin{aligned}
& \dot{z}=f(z)+g(z) \alpha+p(z) d, \\
& y=h(z)
\end{aligned},
$$

where $z \in \mathbb{R}^{n}$ is the state. Suppose that the relative degree of the disturbance $d \in \mathbb{R}$ is equal to one, and that the relative degree of the control $\alpha \in \mathbb{R}$ is equal to two. In symbols,

$$
L_{p} h \neq 0, \quad L_{g} h=0 \quad \text { and } \quad L_{g} L_{f} h \neq 0
$$

with $L_{f} h$ the Lie derivative of the function $h$ along the vector field $f$.

Take $x_{1}=h(z)$ and $x_{2}=L_{f} h(z)$. We have

$$
\begin{aligned}
& \dot{x}_{1}=x_{2}+L_{p} h \cdot d \\
& \dot{x}_{2}=L_{f}^{2} h+L_{g} L_{f} h \cdot \alpha+L_{p} L_{f} h \cdot d .
\end{aligned}
$$

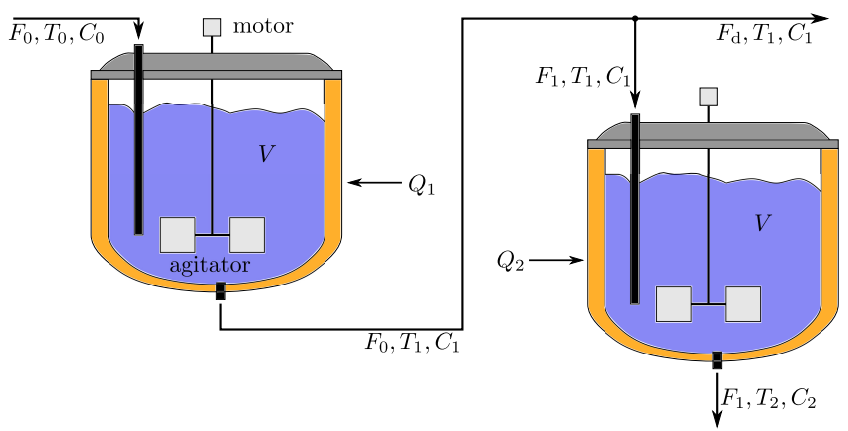

Fig. 1. Two continuously stirred-tank reactors in series.

It is well known that there exist a coordinate transformation $z=\phi(\eta, x)$ such that the dynamics of $\eta$, the internal dynamics, are independent of $\alpha$ [31]. If, furthermore, the internal dynamics are stable, then they can be ignored for the purposes of regulating $y$.

Define the input-output linearizing controller

$$
\alpha=\frac{u-L_{f}^{2} h}{L_{g} L_{f} h}
$$

with $u$ a new control input. The simplified model (10) is then recovered by identifying

$$
\zeta_{1}=L_{p} h \cdot d \quad \text { and } \quad \zeta_{2}=L_{p} L_{f} h \cdot d
$$

\subsection{Example, a chemical process}

Consider two continuously stirred-tank reactors in series, as shown in Figure 1. A reactant enters the first tank at a rate $F_{0}$, a temperature $T_{0}$ and a concentration $C_{0}$. The reactant leaves the tank at the same rate and enters the second one at a temperature $T_{1}$ and a concentration $C_{1}$. A fraction of the stream, $F_{\mathrm{d}}=F_{0} d$, is diverted from $F_{0}$ and used, e.g., at a later stage. The fraction $0 \leq d \leq 1$ is unknown. The remaining flow, $F_{1}=F_{0}(1-d)$, enters the second tank and leaves it at a concentration $C_{2}$ and a temperature $T_{2}$. The volumes of the tanks remain constant and for simplicity we assume that they are both equal to $V$. A simple mass balance together with Arrhenius equation (to account for the temperature dependence of the reaction rates) gives [32]

$\frac{\mathrm{d} C_{1}}{\mathrm{~d} t}=\frac{F_{0}}{V}\left(C_{0}-C_{1}\right)-k_{0} e^{-\frac{E}{R T_{1}}} C_{1}$
$\frac{\mathrm{d} C_{2}}{\mathrm{~d} t}=\frac{F_{0}}{V}\left(C_{1}-C_{2}\right)-k_{0} e^{-\frac{E}{R T_{2}}} C_{2}-\frac{F_{0}}{V}\left(C_{1}-C_{2}\right) \cdot d$,

where $k_{0}$ is the pre-exponential factor, $E$ is the activation energy and $R$ is the universal gas constant. Energy 
balance gives

$$
\begin{aligned}
\frac{\mathrm{d} T_{1}}{\mathrm{~d} t}= & \frac{F_{0}}{V}\left(T_{0}-T_{1}\right)-\frac{\Delta H}{\rho c_{p}} k_{0} e^{-\frac{E}{R T_{1}}} C_{1}+\frac{Q_{1}}{V \rho c_{p}} \\
\frac{\mathrm{d} T_{2}}{\mathrm{~d} t}= & \frac{F_{0}}{V}\left(T_{1}-T_{2}\right)-\frac{\Delta H}{\rho c_{p}} k_{0} e^{-\frac{E}{R T_{2}}} C_{2}+\frac{Q_{2}}{V \rho c_{p}} \\
& \quad-\frac{F_{0}}{V}\left(T_{1}-T_{2}\right) \cdot d,
\end{aligned}
$$

where $\rho$ is the density of the substance, $c_{p}$ the heat capacity, $\Delta H$ is the heat of the reaction and $Q_{1}$ and $Q_{2}$ are the heat inputs in the first and second vessels, respectively. The objective is to regulate, by acting on $Q_{2}$, the effluent concentration $C_{2}$ to the desired value $C_{2 \mathrm{~d}}$.

The state, input and output are, respectively,

$$
\begin{aligned}
& z=\left(\begin{array}{llll}
C_{1} & C_{2} & T_{1} & T_{2}
\end{array}\right)^{\top}, \quad \alpha=Q_{2} \quad \text { and } \\
& y=h(z)=z_{2}-C_{2 \mathrm{~d}} .
\end{aligned}
$$

The vector fields are

$$
\begin{gathered}
f(z)=\left(\begin{array}{c}
\frac{F_{0}}{V}\left(C_{0}-z_{1}\right)-k_{0} e^{-\frac{E}{R z_{3}}} z_{1} \\
\frac{F_{0}}{V}\left(z_{1}-z_{2}\right)-k_{0} e^{-\frac{E}{R z_{4}}} z_{2} \\
\frac{F_{0}}{V}\left(T_{0}-z_{3}-\frac{\Delta H}{\rho c_{p}} k_{0} e^{-\frac{E}{R z_{3}}} z_{1}+\frac{Q_{1}}{V \rho c_{p}}\right. \\
\frac{F_{0}}{V}\left(z_{3}-z_{4}\right)-\frac{\Delta H}{\rho c_{p}} k_{0} e^{-\frac{E}{R z_{4}}} z_{2}
\end{array}\right), \\
p(z)=\left(\begin{array}{c}
0 \\
\frac{F_{0}}{V}\left(z_{2}-z_{1}\right) \\
0 \\
\frac{F_{0}}{V}\left(z_{4}-z_{3}\right)
\end{array}\right) \text { and } g(z)=\left(\begin{array}{l}
0 \\
0 \\
1
\end{array}\right) .
\end{gathered}
$$

It is lengthy but straightforward to verify that (11) holds. Also, by setting

$$
x_{1}=z_{2}-C_{2 \mathrm{~d}}, \quad x_{2}=\frac{F_{0}}{V}\left(z_{1}-z_{2}\right)-k_{0} e^{-\frac{E}{R z_{4}}} z_{2}
$$

and the control (12) with

$$
\begin{gathered}
L_{f}^{2} h=\frac{E k_{0} z_{2}}{R z_{4}^{2}}\left(\frac{\Delta H k_{0}}{\rho c_{p}} z_{2} e^{-\frac{E}{R z_{4}}}-\frac{F_{0}}{V}\left(z_{3}-z_{4}\right)\right) . \\
e^{-\frac{E}{R z_{4}}}+\frac{F_{0}}{V}\left(\frac{F_{0}}{V}\left(C_{0}-z_{1}\right)-k_{0} z_{1} e^{-\frac{E}{R z_{3}}}\right) \\
-\left(\frac{F_{0}}{V}+k_{0} e^{-\frac{E}{R z_{4}}}\right)\left(\frac{F_{0}}{V}\left(z_{1}-z_{2}\right)-k_{0} z_{2} e^{-\frac{E}{R z_{4}}}\right)
\end{gathered}
$$

and

$$
L_{g} L_{f} h=-\frac{E k_{0} z_{2} e^{-\frac{E}{R z_{4}}}}{R V \rho z_{4}^{2}},
$$

one obtains (10) with

$$
\zeta_{1}=\frac{F_{0}}{V}\left(z_{2}-z_{1}\right) \cdot d
$$

and

$$
\begin{aligned}
\zeta_{2}=\frac{F_{0} e^{-\frac{E}{R z_{4}}}}{R V^{2} z_{4}^{2}} & \left(E V k_{0} z_{2}\left(z_{3}-z_{4}\right)\right. \\
& \left.+R z_{4}^{2}\left(z_{1}-z_{2}\right)\left(F_{0} e^{\frac{E}{R z_{4}}}+V k_{0}\right)\right) \cdot d .
\end{aligned}
$$

\section{Continuous-time nested set-valued control}

In order to achieve the robust regulation of $y$ to a neighbourhood of the origin, we propose the control law

$$
u(x) \in-\gamma_{2} \Xi(x)-\gamma_{3} \operatorname{Sgn}(\Xi(x)),
$$

where $\Xi: \mathbb{R}^{2} \rightrightarrows \mathbb{R}$ is the set valued map $x \mapsto x_{2}+$ $\gamma_{1} \operatorname{Sgn}\left(x_{1}\right)$ and the gains $\gamma_{i}, i=1,2,3$, are positive and constant. The set $\{x \mid 0 \in \Xi(x)\}$ may be thought of as a nonsmooth switching surface. The composed multifunction $\operatorname{Sgn}\left(x_{2}+\gamma_{1} \operatorname{Sgn}\left(x_{1}\right)\right)$ is computed from (1) as

$$
\operatorname{Sgn}(\Xi(x))=\left\{\begin{array}{ll}
\operatorname{sgn}(\xi(x)) & \text { if } x_{1} \neq 0,0 \notin \Xi(x) \\
\operatorname{sgn}\left(x_{2}\right) & \text { if } x_{1}=0,0 \notin \Xi(x) \\
{[-1,1]} & \text { if } x_{1} \in \mathbb{R}, 0 \in \Xi(x)
\end{array},\right.
$$

where $\xi:\left\{x \in \mathbb{R}^{2} \mid x_{1} \neq 0\right\} \rightarrow \mathbb{R}$ is a singled-valued map given as

$$
\xi(x)=x_{2}+\gamma_{1} \operatorname{sgn}\left(x_{1}\right)
$$

(see Figure 2).

Since the disturbances $\zeta_{i}$ are uniformly bounded (see Assumption 9) it is always possible to find gains $\gamma_{1}$ and $\gamma_{3}$ sufficiently large such that the following holds:

i) $\zeta_{1}\left(t, x_{1}\right) \in \gamma_{1} \cdot \mathcal{B}_{1}$, for all $t \geq 0$,

ii) $\zeta_{2}(t, x) \in \gamma_{3} \cdot \mathcal{B}_{1}$, for all $t \geq 0$.

With the interconnection of (14) and (10), the closedloop system can be modeled by the differential inclusion

$$
\begin{aligned}
\dot{x}_{1} & =x_{2}+\zeta_{1} \\
\dot{x}_{2} & \in-\gamma_{2} \Xi(x)-\gamma_{3} \operatorname{Sgn} \Xi(x)+\zeta_{2} . \\
y & =x_{1}
\end{aligned}
$$

A pair of typical trajectories is shown in Figure 3. They consist of an initial reaching phase, a sliding motion along a continuous segment of the switching surface and oscillations around the corners of the switching surface. 


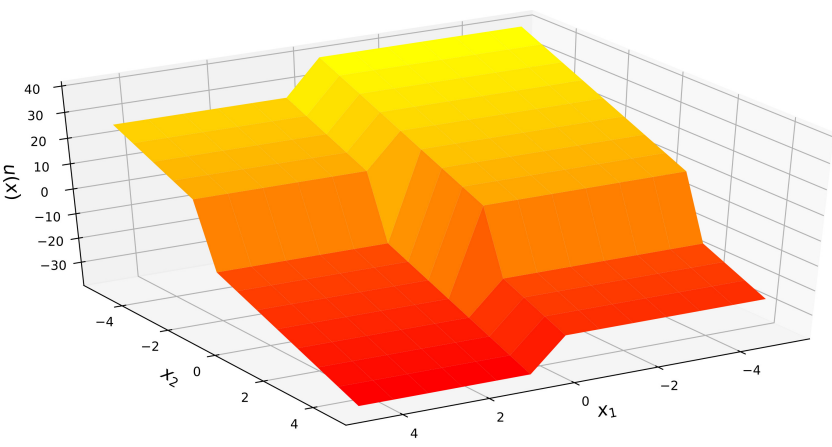

Fig. 2. Control $u(x)$ (on vertical axis) defined by (14) with $\gamma_{1}=1.5, \gamma_{2}=4.5$ and $\gamma_{3}=10.75$.

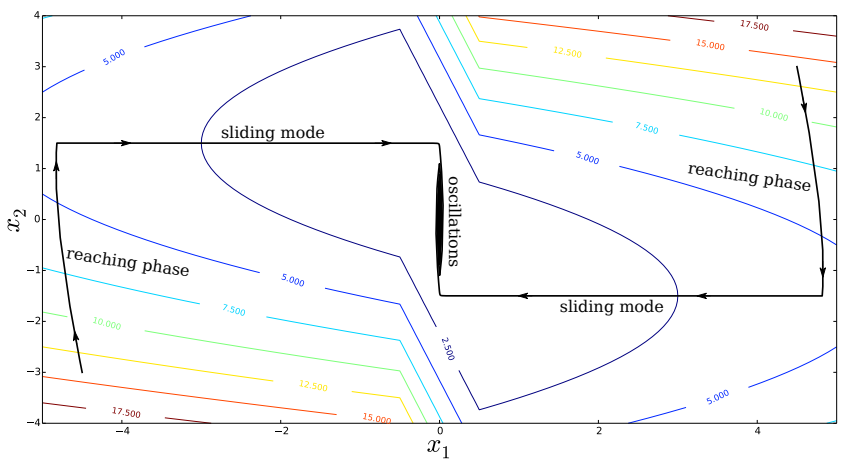

Fig. 3. Closed-loop trajectories of (16) (lines with arrows) and level sets (23) of the nonsmooth Lyapunov function (22). The system parameters are $\zeta_{1}\left(t, x_{1}\right)=-\sin \left(x_{1}\right)$, $\zeta_{2}(t, x) \equiv 0, \gamma_{1}=1.5, \gamma_{2}=4.5$ and $\gamma_{3}=10.75$.

In the last mode, the swings along the $x_{2}$ axis are relatively large. This is to be expected as $x_{2}$ is required to compensate for $\zeta_{1}$, according to the backstepping-like control strategy. On the other hand, the amplitude of $x_{1}$ is rather small, as desired. The formal analysis of such behaviour is the topic of the sequel.

\subsection{Well-posedness of the closed-loop}

Proposition 10 Consider the closed-loop system (16) and define the set-valued map

$$
\mathbf{G}(t, x):=\left(\begin{array}{c}
x_{2}+\zeta_{1}\left(t, x_{1}\right) \\
-\gamma_{2} \Xi(x)-\gamma_{3} \operatorname{Sgn}(\Xi(x))+\zeta_{2}(t, x)
\end{array}\right),
$$

where the perturbations $\zeta_{i}$ satisfy Assumption 9. There exist $T>0$ and an absolutely continuous function $x(\cdot)$ defined on $[0, T]$, a solution to the differential inclusion (2).

PROOF. It suffices to verify the conditions of Theorem 3. It follows from [33, Proposition 2.2] that the setvalued function Sgn is usc. By Proposition 2 and the continuity of $\zeta_{i}$ with respect to $x$, the set-valued map (17) is then usc. Since its values consist of sums of closed bounded intervals, such values are convex and compact. Also, the map $(t, x) \mapsto \mathbf{G}(t, x)$ is locally compact.

Remark 11 It is shown later in Section 4.2 that all solutions remain bounded. Using standard arguments, it is then possible to show that $T$ can be chosen arbitrarily large. Alternatively, the global existence of solutions can be established by invoking [34, Theorem 5.2], which is formulated in the much broader context of Marchaud differential inclusions.

Proposition 10 assures the existence of solutions for any initial condition. However, the uniqueness of solutions requires further properties which we do not investigate here, keeping in mind that the stability proofs in the sequel accommodate non-uniqueness of solutions.

We also remark that, because of the presence of the mismatched uncertain terms, it is in general impossible to drive the whole state to the origin. In fact, system (10) does not contain an assignable equilibrium at the origin, unless $\zeta_{1}$ vanishes at the origin. Thus, we aim for stability of a set rather than just a point.

\subsection{Ultimate boundedness of the closed loop}

Let us start by introducing two single-valued mappings associated with the set-valued sign function,

$$
\begin{aligned}
J_{\mathrm{S}}^{\varepsilon} & =(I+\varepsilon \operatorname{Sgn})^{-1} \\
\mathcal{S}^{\varepsilon} & =\frac{1}{\varepsilon}\left(I-J_{\mathbf{S}}^{\varepsilon}\right) .
\end{aligned}
$$

which are the so-called resolvent and Yosida approximation of Sgn, respectively. It follows from these definitions that

$$
\begin{aligned}
& J_{\mathbf{S}}^{\varepsilon}(x)= \begin{cases}x-\varepsilon \operatorname{sgn}(x) & \text { if }|x|>\varepsilon \\
0 & \text { if }|x| \leq \varepsilon\end{cases} \\
& \mathcal{S}^{\varepsilon}(x)= \begin{cases}\operatorname{sgn}(x) & \text { if }|x|>\varepsilon \\
\frac{x}{\varepsilon} & \text { if }|x| \leq \varepsilon\end{cases}
\end{aligned}
$$

Clearly, $\mathcal{S}^{\varepsilon}$ is Lipschitz continuous with constant $1 / \varepsilon$, while $J_{\mathbf{S}}^{\varepsilon}$ is non-expansive, i.e., it is a Lipschitz continuous map with constant less or equal than one (see [35] for more details). Note also that $\varepsilon \mathcal{S}^{\varepsilon}(x) \in \operatorname{Proj}(x,[-\varepsilon, \varepsilon])$.

Now we are ready to prove the ultimate boundedness of the state of the closed-loop system (16). We will do so by using the nonsmooth, Lipschitz continuous, regular and proper Lyapunov function

$$
V\left(x_{1}, x_{2}\right)=\left|x_{1}\right|_{[-\varepsilon, \varepsilon]}+\frac{1}{2}\left(x_{2}+\gamma_{1} \mathcal{S}^{\varepsilon}\left(x_{1}\right)\right)^{2}
$$


Note that this function is nonnegative everywhere and equal to zero only on the line segment joining the points $\left(-\varepsilon, \gamma_{1}\right)$ and $\left(\varepsilon,-\gamma_{1}\right)$. Figure 3 shows the level sets

$$
\mathcal{V}_{c}:=\left\{x \in \mathbb{R}^{2} \mid V(x)=c\right\}
$$

for a particular instance of such function.

Theorem 12 Consider the closed-loop system (16). Fix $\varepsilon>0$ and define $\Omega_{c^{*}}$, the smallest sublevel set containing the rectangle $\mathcal{Q}:=[-\varepsilon, \varepsilon] \times\left[-\gamma_{1}, \gamma_{1}\right]$. In other words, set

$$
c^{*}=\min \left\{c \mid \mathcal{Q} \subseteq \Omega_{c}\right\} .
$$

The set $\Omega_{c^{*}}$ is globally asymptotically stable (this implies that the system trajectories are ultimately bounded) whenever

$$
\begin{aligned}
& \gamma_{1} \geq w_{1} \\
& \gamma_{2} \geq \frac{\gamma_{1}}{\varepsilon}, \\
& \gamma_{3} \geq w_{2}+\max \left\{1, \gamma_{1}\left(\gamma_{2}+\frac{1}{\varepsilon} w_{1}\right)\right\} .
\end{aligned}
$$

Before stating the proof, allow us to define the autonomous set-valued map

$$
\mathbf{F}(x):=\left(\begin{array}{c}
x_{2}+w_{1} \mathcal{B}_{1} \\
-\gamma_{2} \Xi(x)-\gamma_{3} \operatorname{Sgn}(\Xi(x))+w_{2} \mathcal{B}_{1}
\end{array}\right) .
$$

A short comment on the use of (17) and (25) as models for (16) is in order. Note that the formulation with $F(x)$ is more general than the formulation with $G(t, x)$, as $F(x)$ does not impose any assumptions on the continuity nor the measurably of the perturbations, it just requires boundedness. The non-autonomous map $G(t, x)$ was used in Proposition 10, because the existence of one solution for $F(x)$ would merely imply that a solution exists for one particular perturbation. This would of course be undesirable, as solutions should exist for any perturbation in the given class. Now, we use the map $F(x)$ to prove stability because the tools we use require the system to be autonomous. The mathematical argument is correct in as much as Theorem 8 holds for all solutions. Thus, what is proved below for (25) is actually stronger than what is stated for (16).

PROOF. As first step we compute the generalized gradient of the distance function to the closed set $\mathcal{C}:=$ $[-\varepsilon, \varepsilon]$ as indicated in $[36$, Theorem 1$]$,

$$
\partial|x|_{\mathcal{C}}= \begin{cases}\operatorname{sgn}(x) & \text { if }|x|>\varepsilon \\ {[0,1] \operatorname{sgn}(x)} & \text { if }|x|=\varepsilon \\ 0 & \text { if }|x|<\varepsilon\end{cases}
$$

On the other hand, it is easy to see that the generalized gradient of the Yosida approximation of Sgn exists and satisfies

$$
\partial \mathcal{S}^{\varepsilon}(x)= \begin{cases}0 & \text { if }|x|>\varepsilon \\ {\left[0, \frac{1}{\varepsilon}\right]} & \text { if }|x|=\varepsilon . \\ \frac{1}{\varepsilon} & \text { if }|x|<\varepsilon\end{cases}
$$

Therefore, following the nonsmooth chain rule in Proposition 7, along with (26)-(27) and (15), we compute the generalized gradient of $(22)$,

$$
\begin{aligned}
& \partial V(x)= \\
& \begin{cases}\left(\operatorname{sgn}\left(x_{1}\right), \xi(x)\right) & \text { if }\left|x_{1}\right|>\varepsilon \\
\left([0,1]\left(\operatorname{sgn}\left(x_{1}\right)+\frac{\gamma_{1}}{\varepsilon} \xi(x)\right), \xi(x)\right) & \text { if }\left|x_{1}\right|=\varepsilon \\
\left(\frac{\gamma_{1}}{\varepsilon}\left(x_{2}+\frac{\gamma_{1}}{\varepsilon} x_{1}\right), x_{2}+\frac{\gamma_{1}}{\varepsilon} x_{1}\right) & \text { if }\left|x_{1}\right|<\varepsilon\end{cases}
\end{aligned}
$$

Now we compute the set-valued derivative of $V$ along the trajectories of (2). We analyze three cases that cover the complement of $\mathcal{Q}$.

Case $1\left(\left|x_{1}\right|>\varepsilon\right)$. Note that $\partial V$ is single valued, so from Remark 4 and (28) we obtain

$$
\begin{aligned}
\mathcal{L}_{\mathbf{F}} V(x)= & \operatorname{sgn}\left(x_{1}\right)\left(x_{2}+w_{1} \cdot \mathcal{B}_{1}\right)+ \\
& \xi(x)\left(-\gamma_{2} \xi(x)-\gamma_{3} \operatorname{Sgn}(\xi(x))+w_{2} \cdot \mathcal{B}_{1}\right) .
\end{aligned}
$$

It follows from $x_{2}=\xi(x)-\gamma_{1} \operatorname{sgn}\left(x_{1}\right)$ that

$$
\max \mathcal{L}_{\mathbf{F}} V(x) \leq-W_{1}(x)
$$

with

$$
W_{1}(x)=\left(\gamma_{1}-w_{1}\right)+\gamma_{2} \xi(x)^{2}+\left(\gamma_{3}-w_{2}-1\right)|\xi(x)|,
$$

which is strictly negative whenever $\gamma_{1}>w_{1}, \gamma_{2}>0$ and $\gamma_{3}>1+w_{2}$.

Case $2\left(\left|x_{1}\right|=\varepsilon\right.$ and $\left.\left|x_{2}\right|>\gamma_{1}\right)$. Note first that the condition $\left|x_{2}\right|>\gamma_{1}$ ensures that $\xi(x) \neq 0$. We have

$$
\begin{aligned}
& \mathcal{L}_{\mathbf{F}} V(x)=\left\{a \in \mathbb{R} \mid \exists \zeta_{1} \in w_{1} \cdot \mathcal{B}_{1}, \zeta_{2} \in w_{2} \cdot \mathcal{B}_{2}\right. \\
& \text { such that }\left(\operatorname{sgn}\left(x_{1}\right)+\frac{\gamma_{1}}{\varepsilon} \xi(x)\right)\left(x_{2}+\zeta_{1}\right)[0,1] \\
& \left.\quad+\xi(x)\left(-\gamma_{2} \xi(x)-\gamma_{3} \operatorname{sgn}(\xi(x))+\zeta_{2}\right)=\{a\}\right\} .
\end{aligned}
$$

For the equality inside the braces to hold it is required that

$$
\operatorname{sgn}\left(x_{1}\right)+\frac{\gamma_{1}}{\varepsilon} \xi(x)=0 \quad \text { or } \quad x_{2}+\zeta_{1}=0
$$

in which case we have $a=-\gamma_{2} \xi(x)^{2}-\gamma_{3}|\xi(x)|+\zeta_{2} \xi(x)$. Thus,

$$
\mathcal{L}_{\mathbf{F}} V(x)=-\gamma_{2} \xi(x)^{2}-\left(\gamma_{3}-w_{2} \cdot \mathcal{B}_{1}\right)|\xi(x)| .
$$


Noting that, in this case $|\xi(x)| \geq|x|_{\mathcal{Q}}$, we can write

$$
\mathcal{L}_{\mathrm{F}} V(x) \leq-W_{2}(x)
$$

with

$$
W_{2}(x)=\gamma_{2}|x|_{\mathcal{Q}}^{2}+\left(\gamma_{3}-w_{2}\right)|x|_{\mathcal{Q}},
$$

where (24c) is used to establish the positivity of the coefficient $\gamma_{3}-w_{2}$.

Case $3\left(\left|x_{1}\right|<\varepsilon\right.$ and $\left.\left|x_{2}\right|>\gamma_{1}\right)$. Note that $\partial V$ is again single valued, so

$$
\begin{aligned}
\mathcal{L}_{\mathbf{F}} V(x)=\frac{\gamma_{1}}{\varepsilon}\left(x_{2}+\gamma_{1} \mathcal{S}^{\varepsilon}\left(x_{1}\right)\right)\left(x_{2}+w_{1} \cdot \mathcal{B}_{1}\right) \\
+\left(x_{2}+\gamma_{1} \mathcal{S}^{\varepsilon}\left(x_{1}\right)\right)\left(-\gamma_{2}\left(x_{2}+\gamma_{1} \operatorname{Sgn}\left(x_{1}\right)\right)\right. \\
\left.\quad-\gamma_{3} \operatorname{sgn}\left(x_{2}+\gamma_{1} \operatorname{Sgn}\left(x_{1}\right)\right)+w_{2} \cdot \mathcal{B}_{1}\right),
\end{aligned}
$$

where we have used $\mathcal{S}^{\varepsilon}\left(x_{1}\right)=x_{1} / \varepsilon$.

Since

$$
\operatorname{sgn}\left(x_{2}+\gamma_{1} \operatorname{Sgn}\left(x_{1}\right)\right)=\operatorname{sgn}\left(x_{2}\right)
$$

and

we can write

$$
\operatorname{sgn}\left(x_{2}+\gamma_{1} \mathcal{S}^{\varepsilon}\left(x_{1}\right)\right)=\operatorname{sgn}\left(x_{2}\right),
$$

$$
\begin{array}{r}
\mathcal{L}_{\mathbf{F}} V(x)=-\left|x_{2}+\gamma_{1} \mathcal{S}^{\varepsilon}\left(x_{1}\right)\right|\left|x_{2}\right|\left(\gamma_{2}-\frac{\gamma_{1}}{\varepsilon}\right) \\
-\left|x_{2}+\gamma_{1} \mathcal{S}^{\varepsilon}\left(x_{1}\right)\right|\left(\gamma_{3}+\gamma_{1} \gamma_{2} \operatorname{sgn}\left(x_{2}\right) \operatorname{Sgn}\left(x_{1}\right)\right) \\
\left.-\frac{\gamma_{1}}{\varepsilon} w_{1} \operatorname{sgn}\left(x_{2}\right) \cdot \mathcal{B}_{1}+w_{2} \cdot \mathcal{B}_{1}\right) .
\end{array}
$$

Therefore,

$$
\begin{aligned}
\max & \mathcal{L}_{\mathbf{F}} V(x) \leq-\left|x_{2}+\gamma_{1} \mathcal{S}^{\varepsilon}\left(x_{1}\right)\right| \cdot \\
& \left(\left|x_{2}\right|\left(\gamma_{2}-\frac{\gamma_{1}}{\varepsilon}\right)+\gamma_{3}-\gamma_{1} \gamma_{2}-\frac{\gamma_{1}}{\varepsilon} w_{1}-w_{2}\right) .
\end{aligned}
$$

In this case we have $\left|x_{2}+\gamma_{1} \mathcal{S}^{\varepsilon}\left(x_{1}\right)\right| \geq|x|_{\mathcal{Q}}$, so we can write $\mathcal{L}_{\mathbf{F}} V(x) \leq-W_{3}(x)$ with

$$
\begin{aligned}
W_{3}(x)=|x|_{\mathcal{Q}} \cdot\left(\left|x_{2}\right|\right. & \left(\gamma_{2}-\frac{\gamma_{1}}{\varepsilon}\right) \\
& \left.+\gamma_{3}-\gamma_{1} \gamma_{2}-\frac{\gamma_{1}}{\varepsilon} w_{1}-w_{2}\right) .
\end{aligned}
$$

We can finally establish (6) with

$$
W(x)=\min \left\{W_{1}(x), W_{2}(x), W_{3}(x)\right\} .
$$

By construction, the function $W$ inherits the continuity and positive definiteness of $W_{i}, i=1,2,3$. We conclude, in accordance with Theorem 8 , the stability of $\Omega_{c^{*}}$ and the ultimate boundedness of the trajectories of (16).

$$
\begin{array}{ll}
E=76,480[\mathrm{~J} / \mathrm{gmol}] & \rho c_{p}=30,000[\mathrm{~J} / \mathrm{L} \cdot \mathrm{K}] \\
V=9[\mathrm{~L}] & \Delta H=-500 \times 10^{3}[\mathrm{~J} / \mathrm{gmol}] \\
R=8.314[\mathrm{~J} / \mathrm{gmol} \cdot \mathrm{K}] & k_{0}=1.25 \times 10^{1} 4
\end{array}
$$

Table 1

System parameters for the chemical reactor.

Remark 13 The width of the set $\mathcal{Q}$ can be made arbitrary small (at the cost of increasing the gains $\gamma_{2}$ and $\left.\gamma_{3}\right)$. Hence, the regulation of the output $y=x_{1}$ can be achieved, in theory, with an arbitrary precision. In practice, however, there exist bounds on the magnitude of the gains, which will restrict the actual precision of the controlled system.

\subsection{Example, a chemical process (continued)}

The chemical process described in Section 3 was simulated with three controllers:

\section{Sliding-mode control $u \in-40 \operatorname{Sgn}\left(5 x_{1}+x_{2}\right)$ \\ H-infinity control $u=-70.43 x_{1}-24.38 x_{2}$ Nested control}

$$
u \in-10 \operatorname{Sgn}\left(0.5 \operatorname{Sgn}\left(x_{1}\right)+x_{2}\right)-10 \cdot\left(0.5 \operatorname{Sgn}\left(x_{1}\right)+x_{2}\right),
$$

all three applied after feedback linearization. The second controller was obtained by minimizing the H-infinity norm of the transfer matrix from the disturbance $\left(\zeta_{1}, \zeta_{2}\right)$ to the virtual output $\left(10 x_{1}, u\right)$. Note that, in a manner consistent with our problem statement, most of the weight of the virtual output is attributed to $x_{1}$, i.e., $x_{2}$ is not present and $u$ appears with a relatively low weight, only to ensure that the control problem is well posed. The linear control gain was computed by solving the corresponding Riccati equation.

The system parameters, taken from [32], are shown in Table 1 . The objective was to regulate the concentration $C_{2}$ to the reference $C_{2 \mathrm{~d}}=0.2$. The incoming flow was set at $F_{0}=54[\mathrm{~L} / \mathrm{h}]$. The time-varying parameters are depicted in Figure 4 . The regulation errors and control actions are shown in Figures 5 and 6, respectively. The smallest steady-state error results for the nested controller. For reference, the solutions are shown on the $x_{1}-x_{2}$ plane (Figure 7 ), where the reaching, the sliding and the oscillating phases of the nested controller can be appreciated, as well as the fact that $x_{1}$ is ultimately bounded to a smaller region. This comparison analysis demonstrates that the nested controller may possess quite interesting properties, and is worth investigating further.

\section{Discrete-time nested set-valued control}

In this section we study the discrete-time counterpart of the nested controller introduced in Section 4. It has been shown in $[15,17,19,20,12]$ that the adequate selection of 

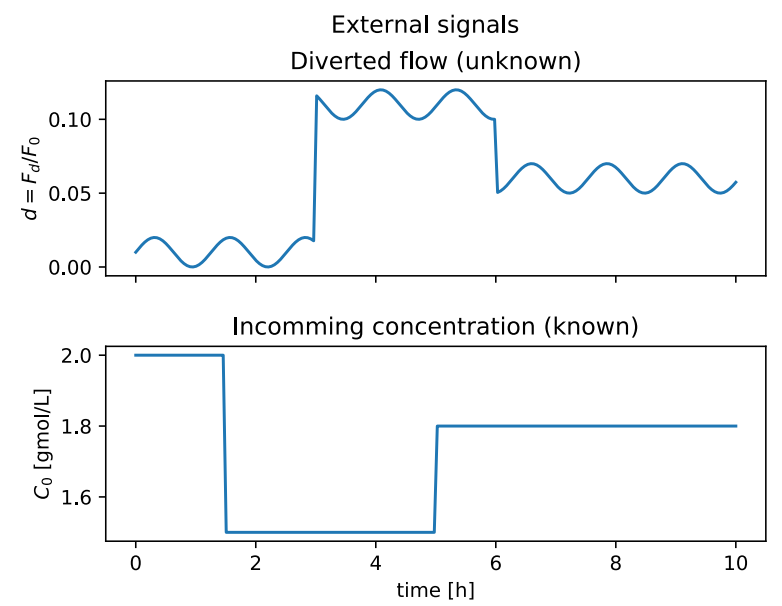

Fig. 4. Time-varying parameters of the chemical process

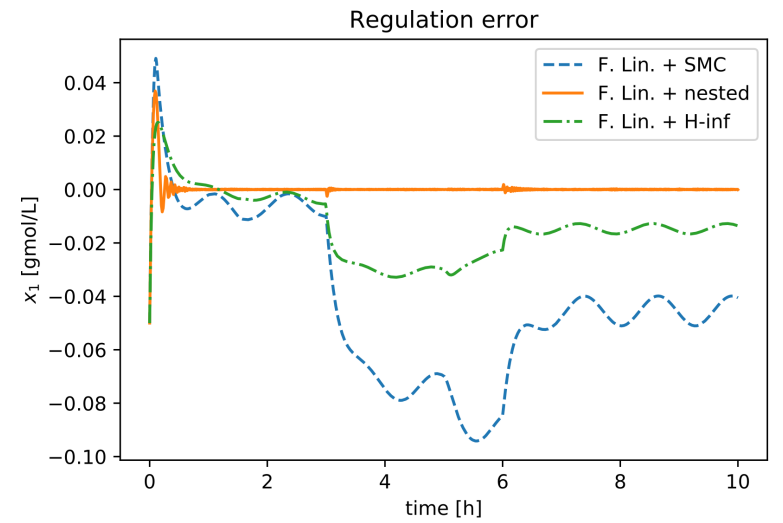

Fig. 5. Regulation error for various control laws applied to the chemical reactors.

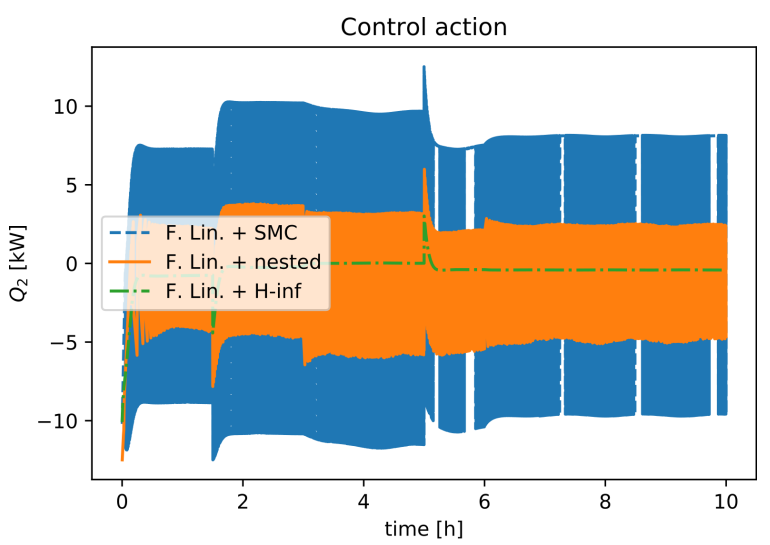

Fig. 6. Various control laws applied to the chemical reactors.

the values of a set-valued controller can substantially reduce the chattering effect. The main contributions of the aforementioned works rely on an implicit discretization scheme which, by using a nominal model of the closed loop, makes the selection of the values of the controller such that matched (or mismatched but vanishing) disturbances are compensated. The main theoretical assumption depends on the maximal monotonicity of the

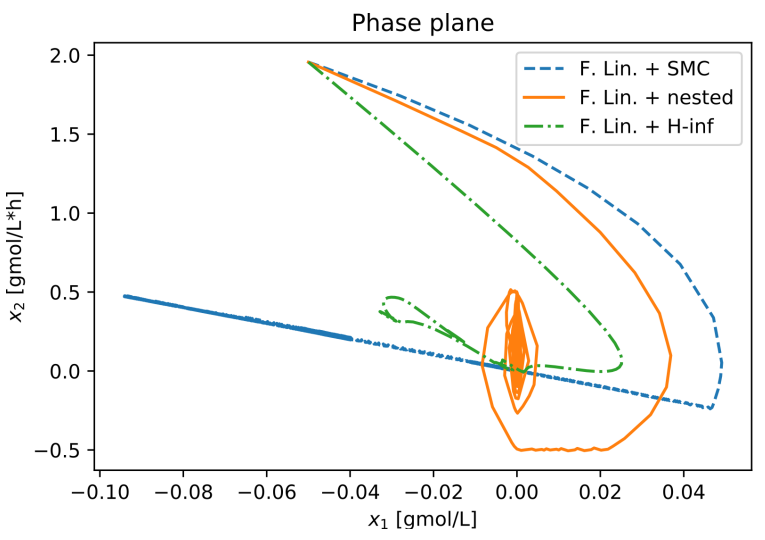

Fig. 7. Phase plane of the feedback-linearized chemical reactors. Various control laws applied.

set-valued map defining the controller, which translates into a single-valued expression in terms of the current state [12]. It is worth mentioning that, in the case when the controller is not a maximal monotone operator, it is not possible (in general) to apply the results previously reported in the literature.

Roughly speaking, we will exploit the triangular structure (10) by applying a backstepping-like algorithm. The algorithms reported in $[15,19,12]$ will be then used to yield a suitable discrete-time control law that exhibits much lower digital chattering than the 'conventional' explicit Euler discretization.

Consider the following discrete model for (10):

$$
\begin{aligned}
x_{1, k+1} & =x_{1, k}+h\left(x_{2, k+1}+\zeta_{1, k}\right) \\
x_{2, k+1} & =x_{2, k}+h\left(u_{k}+\zeta_{2, k}\right) \\
y_{k} & =x_{1, k},
\end{aligned}
$$

where $h=t_{k+1}-t_{k}>0$ represents the sampling period, considered fixed, and the notation $f_{i, k}$ stands for $f_{i}\left(t_{k}\right)$.

Now we go one step through the backstepping-like algorithm. We consider the virtual system

$$
x_{1, k+1}=x_{1, k}+h \nu_{k}+h \zeta_{1, k}
$$

with virtual control input $\nu_{k}$ and we consider its nominal version

$$
\tilde{x}_{1, k+1}=x_{1, k}+h \nu_{k} \text {. }
$$

We know that the set-valued control law

$$
\nu_{k} \in-\gamma_{1} \operatorname{Sgn}\left(\tilde{x}_{1, k+1}\right)
$$

is well posed. In the discrete context, by well posed we mean that, for any given sequence $x_{1, k}$ :

i) There exists sequences $\tilde{x}_{1, k}$ and $\nu_{k}$ satisfying (30) and (31) for all $k \in \mathbb{N}$. 
ii) There is a unique selection for the control.

By item ii) we mean that, given $x_{1, k}$ at some $k \in \mathbb{N}$, there is one, and only one, $\nu_{k}$ that satisfies (30) and (31) simultaneously.

We also know that (30) and (31) allow for a suitable selection strategy that achieves the robust regulation of the virtual state $\tilde{x}_{1, k}$ to the origin in finite time for $\gamma_{1}>0$ sufficiently large, and that the system admits the Lyapunov function $V_{1}\left(x_{1}\right)=\left|x_{1}\right|$ (see, e.g., $[15,19,12]$ ). It is worth recalling that steering $\tilde{x}_{1, k}$ towards the origin implies that $\left|x_{1, k}\right| \leq h w_{1}$. Then we have the limit $x_{1, k} \rightarrow$ 0 as $h \rightarrow 0$.

Next, we go one step further into the backstepping algorithm by considering the whole system (29) and the control law

$$
u_{k}=-\gamma_{2}\left(x_{2, k}-\nu_{k}\right)+\eta_{k},
$$

where $\nu_{k}$ is as in (30)-(31) and

$$
\begin{aligned}
\eta_{k} & \in-\gamma_{3} \operatorname{Sgn}\left(\tilde{x}_{2, k+1}-\nu_{k}\right) \\
\tilde{x}_{2, k+1} & =x_{2, k}+h u_{k} .
\end{aligned}
$$

Note that (31)-(33) constitute the forward discretization of (14)-(15), where $\tilde{x}_{k+1}$ is used in place of $x_{k+1}$ because $x_{k+1}$ requires knowledge of $\zeta_{k}$.

Note also that (30)-(31) make the generalized equation

$$
0 \in-\nu_{k}-\gamma_{1} \operatorname{Sgn}\left(x_{1, k}+h \nu_{k}\right)
$$

with unknown $\nu_{k}$, and that (32)-(34) make

$$
0 \in-\gamma_{2}\left(x_{2, k}-\nu_{k}\right)-u_{k}-\gamma_{3} \operatorname{Sgn}\left(x_{2, k}-\nu_{k}+h u_{k}\right)
$$

with unknown $u_{k}$. We now study the well-posedness and stability of the closed-loop system (29)-(34). The use of the backstepping-like approach allows us to overcome the maximal monotonicity requirement by splitting the selection process in two steps. Namely, we first compute the selection of the values of $\nu_{k}$ by using (30)-(31). After that, we use (33)-(34) to compute the selection of $\eta_{k}$, considering $\nu_{k}$ fixed. The following lemma formalizes this.

Lemma 14 The closed-loop system (29)-(34) is well posed. The unique selection of the control depends on the current state $x\left(t_{k}\right)$ only. Moreover, we have

$$
\begin{aligned}
\tilde{x}_{1, k+1} & =J_{\mathbf{S}}^{h \gamma_{1}}\left(x_{1, k}\right) \\
\tilde{x}_{2, k+1}-\nu_{k} & =J_{\mathbf{S}}^{h \gamma_{3}}\left(\left(1-h \gamma_{2}\right)\left(x_{2, k}-\nu_{k}\right)\right),
\end{aligned}
$$

which in fact implies

$$
\begin{aligned}
\nu_{k} & =-\gamma_{1} \mathcal{S}^{h \gamma_{1}}\left(x_{1, k}\right) \\
\eta_{k} & =-\gamma_{3} \mathcal{S}^{h \gamma_{3}}\left(\left(1-h \gamma_{2}\right)\left(x_{2, k}-\nu_{k}\right)\right) .
\end{aligned}
$$

PROOF. Consider the subsystem described by (30)(31). It can be rewritten as

$$
x_{1, k} \in\left(I+h \gamma_{1} \operatorname{Sgn}\right)\left(\tilde{x}_{1, k+1}\right) .
$$

Recalling the definition of the resolvent given by (18), it becomes evident that $\tilde{x}_{1, k+1}=J_{\mathbf{S}}^{h \gamma_{1}}\left(x_{1, k}\right)$. It follows from (30) that

$$
J_{\mathbf{S}}^{h \gamma_{1}}\left(x_{1, k}\right)=x_{1, k}+h \nu_{k},
$$

from where we easily infer (36a) with the aid of (19). Equations (35b) and (36b) follow mutatis mutandis by starting the argument with subsystem (33)-(34).

Therefore, the controller is non-anticipative and can be calculated at $t=t_{k}$ and applied on $\left[t_{k}, t_{k+1}\right)$ using (32), (36) and (21).

Once the well-posedness of the closed loop has been established, we turn to the study of the existence of a discrete-time sliding motion.

Definition 15 We say that the closed-loop system (29)(34) is in discrete-time sliding mode at time $k$ if

$$
\nu_{k} \in\left(-\gamma_{1}, \gamma_{1}\right) \quad \text { and } \quad \eta_{k} \in\left(-\gamma_{3}, \gamma_{3}\right) .
$$

Define the sliding set

$\mathcal{R}=\left\{\left(x_{1, k}, x_{2, k}\right) \in \mathbb{R}^{2} \mid \tilde{x}_{1, k+1}=0, \tilde{x}_{2, k+1}-\nu_{k}=0\right\}$,

and note that the trajectories exhibit a sliding motion whenever $x_{k} \in \mathcal{R}$. Also, by (35) and (20), we have

$$
\begin{aligned}
& \mathcal{R}=\left\{x \in \mathbb{R}^{2}|| x_{1} \mid \leq h \gamma_{1},\right. \\
&\left.\left|x_{2}+\gamma_{1} \mathcal{S}^{h \gamma_{1}}\left(x_{1}\right)\right| \leq \frac{h \gamma_{3}}{1-h \gamma_{2}}\right\}
\end{aligned}
$$

The following lemma states conditions for the existence of a sliding regime.

Lemma 16 Consider the closed-loop system (29)-(34) and fix $0<h<1$. The set $\mathcal{R}$ in (38) is robustly positively 
invariant, i.e., it is invariant in the presence of the disturbances $\zeta_{i, k}$, whenever the gains $\gamma_{i}, i=1,2,3$ satisfy

$$
\begin{aligned}
w_{1}+h w_{2} & <\gamma_{1} \\
1<\gamma_{2} & <\frac{1}{h} \\
2 \gamma_{1}+h w_{2} & <\frac{h \gamma_{3}}{1-h \gamma_{2}} .
\end{aligned}
$$

PROOF. Assume that $\left(x_{1, k}, x_{2, k}\right) \in \mathcal{R}$ for some $k>0$. We will show that $\left(x_{1, k+1}, x_{2, k+1}\right) \in \mathcal{R}$. From equations (29a), (30) and (34) we obtain

$$
x_{1, k+1}=\tilde{x}_{1, k+1}+h\left(\tilde{x}_{2, k+1}-\nu_{k}\right)+h \zeta_{1, k}+h^{2} \zeta_{2, k},
$$

but, according to (37) and (39a),

$$
\left|x_{1, k+1}\right| \leq h\left(w_{1}+h w_{2}\right) \leq h \gamma_{1} \text {. }
$$

On the other hand, from (29b) and (34) it follows that

$$
\begin{aligned}
x_{2, k+1}-\nu_{k+1} & =\tilde{x}_{2, k+1}+h \zeta_{2, k}-\nu_{k+1} \\
& =\tilde{x}_{2, k+1}-\nu_{k}+\nu_{k}-\nu_{k+1}+h \zeta_{2, k},
\end{aligned}
$$

so that

$$
\left|x_{2, k+1}-\nu_{k+1}\right| \leq\left|\nu_{k}-\nu_{k+1}\right|+h w_{2} .
$$

Finally, the bounds $\left|\nu_{k}-\nu_{k+1}\right| \leq 2 \gamma_{1}$ and (39b) imply

$$
\left|x_{2, k+1}-\nu_{k+1}\right| \leq \frac{h \gamma_{3}}{1-h \gamma_{2}}
$$

The following theorem depicts the limiting properties of the backstepping-like selection algorithm.

Theorem 17 Consider the closed-loop dynamics given by (29)-(34). Let all the assumptions of Lemma 16 hold. Then, the region $\mathcal{R}$ in (38) is finite-time stable, that is, for any initial condition $x(0) \in \mathbb{R}^{2}$, the closed-loop system trajectories converge to $\mathcal{R}$ in a finite number of steps.

PROOF. Consider the regular candidate Lyapunov function

$$
V\left(k, x_{k}\right)=\left|\tilde{x}_{1, k+1}\right|+\left|\tilde{x}_{2, k+1}-\nu_{k}\right| .
$$

This function is positive definite with respect to $\mathcal{R}$. Note that proving $\tilde{x}_{1, k+1} \rightarrow 0$ and $\tilde{x}_{2, k+1}-\nu_{k} \rightarrow 0$ is equivalent to proving $\left(x_{1, k}, x_{2, k}\right) \rightarrow \mathcal{R}$. The difference $\Delta V_{k}(x):=V\left(x_{k+1}\right)-V\left(x_{k}\right)$ is

$$
\begin{aligned}
\Delta V_{k}(x)=\left|\tilde{x}_{1, k+2}\right| & -\left|\tilde{x}_{1, k+1}\right| \\
& +\left|\tilde{x}_{2, k+2}-\nu_{k+1}\right|-\left|\tilde{x}_{2, k+1}-\nu_{k}\right|,
\end{aligned}
$$

or

$$
\begin{aligned}
\Delta V_{k}(x)= & \left|J_{\mathbf{S}}^{h \gamma_{1}}\left(x_{1, k+1}\right)\right|-\left|\tilde{x}_{1, k+1}\right| \\
& +\left|J_{\mathbf{S}}^{h \gamma_{3}}\left(\left(1-h \gamma_{2}\right)\left(x_{2, k+1}-\nu_{k+1}\right)\right)\right| \\
& -\left|\tilde{x}_{2, k+1}-\nu_{k}\right| .
\end{aligned}
$$

First we write $x_{k+1}$ as a function of $\tilde{x}_{k+1}$ :

$$
\begin{aligned}
& x_{1, k+1}=\tilde{x}_{1, k+1}+h\left(\tilde{x}_{2, k+1}-\nu_{k}+\zeta_{1, k}\right)+h^{2} \zeta_{2, k} \\
& x_{2, k+1}=\tilde{x}_{2, k+1}+h \zeta_{2, k}
\end{aligned}
$$

and then consider a comprehensive set of cases.

Case $1\left(\left|x_{1, k+1}\right| \geq h \gamma_{1}\right.$ and $\left.\left|x_{2, k+1}-\nu_{k+1}\right| \geq \frac{h \gamma_{3}}{1-h \gamma_{2}}\right)$. Observe that, by (20),

$$
|x| \geq \varepsilon \quad \Longrightarrow \quad\left|J_{\mathbf{S}}^{\varepsilon}(x)\right|=|x|-\varepsilon,
$$

so that

$$
\left|J_{\mathbf{S}}^{h \gamma_{1}}\left(x_{1, k+1}\right)\right|=\left|x_{1, k+1}\right|-h \gamma_{1}
$$

and

$$
\begin{aligned}
& \left|J_{\mathbf{S}}^{h \gamma_{3}}\left(\left(1-h \gamma_{2}\right)\left(x_{2, k+1}-\nu_{k+1}\right)\right)\right|= \\
& \left(1-h \gamma_{2}\right)\left|x_{2, k+1}-\nu_{k+1}\right|-h \gamma_{3}
\end{aligned}
$$

and the difference in $V$ satisfies

$$
\begin{aligned}
& \Delta V_{k}(x)=\left|x_{1, k+1}\right|-h \gamma_{1}-\left|\tilde{x}_{1, k+1}\right| \\
& \quad+\left(1-h \gamma_{2}\right)\left|x_{2, k+1}-\nu_{k+1}\right|-h \gamma_{3}-\left|\tilde{x}_{2, k+1}-\nu_{k}\right| .
\end{aligned}
$$

By applying (42) we can bound the difference as

$$
\begin{aligned}
\Delta V_{k}(x) \leq-h & \left(\gamma_{1}-w_{1}-h w_{2}\right) \\
- & h\left(\gamma_{2}-1\right)\left|\tilde{x}_{2, k+1}-\nu_{k}\right| \\
& -h \gamma_{3}+\left(1-h \gamma_{2}\right)\left(2 \gamma_{1}+h w_{2}\right),
\end{aligned}
$$

where we have made use of $\left|\nu_{k}-\nu_{k+1}\right| \leq 2 \gamma_{1}$.

Case $2\left(\left|x_{1, k+1}\right| \geq h \gamma_{1}\right.$ and $\left.\left|x_{2, k+1}-\nu_{k+1}\right|<\frac{h \gamma_{3}}{1-h \gamma_{2}}\right)$. Equation (44a) still holds. By (20), we have

$$
J_{\mathbf{S}}^{h \gamma_{3}}\left(\left(1-h \gamma_{2}\right)\left(x_{2, k+1}-\nu_{k+1}\right)\right)=0 .
$$

Hence, equation (41) results in

$$
\begin{aligned}
& \Delta V_{k}(x)=\left|x_{1, k+1}\right|-h \gamma_{1}-\left|\tilde{x}_{1, k+1}\right|-\left|\tilde{x}_{2, k+1}-\nu_{k}\right| \\
& \leq-h\left(\gamma_{1}-w_{1}-h w_{2}\right)-(1-h)\left|\tilde{x}_{2, k+1}-\nu_{k}\right|
\end{aligned}
$$


Case $3\left(\left|x_{1, k+1}\right|<h \gamma_{1}\right.$ and $\left.\left|x_{2, k+1}-\hat{\nu}_{k+1}\right| \geq \frac{h \gamma_{3}}{1-h \gamma_{2}}\right)$. Equation (44b) holds and $J_{\mathbf{S}}^{h \gamma_{1}}\left(x_{1, k+1}\right)=0$. Hence,

$$
\begin{aligned}
\Delta V_{k}(x) \leq-\left|\tilde{x}_{1, k+1}\right| & -h \gamma_{2}\left|\tilde{x}_{2, k+1}-\nu_{k}\right|-h \gamma_{3} \\
& +\left(1-h \gamma_{2}\right)\left(2 \gamma_{1}+h w_{2}\right) .
\end{aligned}
$$

Case $4\left(\left|x_{1, k+1}\right|<h \gamma_{1}\right.$ and $\left.\left|x_{2, k+1}-\nu_{k+1}\right|<\frac{h \gamma_{3}}{1-h \gamma_{2}}\right)$. Both resolvents are zero, so

$$
\Delta V_{k}(x)=-\left|\tilde{x}_{1, k+1}\right|-\left|\tilde{x}_{2, k+1}-\nu_{k}\right| .
$$

It is not difficult to verify that the inequalities (39) imply that $\Delta V_{k}(x) \leq 0$ in all cases (45)-(48). Moreover, $\Delta V_{k}(x)$ is bounded away from zero outside $\mathcal{R}$ (Cases 1 $-3)$, so $\mathcal{R}$ is attained in finite time.

\subsection{Example, a chemical process (continued)}

In this section we illustrate the performance of the controller applied to the chemical reactors described in previous sections. The simulations of the closed-loop system (13), (12), (32) were carried out using a zero-order hold as interface between the discrete-time controller and the continuous-time plant.

An advantage of the implicit discretization approach is the possibility to use relatively large sampling periods without deteriorating the performance significantly (this has been confirmed experimentally in, e.g., [20]). Allow us then to set $h=1.8 \mathrm{~min}$ and the gains

$$
\gamma_{1}=0.5, \quad \gamma_{2}=10 \quad \text { and } \quad \gamma_{3}=10 .
$$

Figure 8 shows the time evolution of the regulation error $x_{1}$, of $x_{2}$ and the control input. It can be seen that $x_{2}$ compensates for $\zeta_{1}$ (cf. the perturbation in Figure 4). There is an initial control peak which takes $x$ to the set defined by $0 \in \Xi(x)$. Afterwards, the control compensates for $\zeta_{2}$ while steering $x_{2}$ to the trajectory that compensates for $\zeta_{1}$. There are some additional peaks that correspond to the sudden transitions that $x_{2}$ requires to compensate for $\zeta_{1}$. The overall strategy keeps $x_{1}$ practically at zero.

For comparison purposes, the same plant with the same parameters, was simulated using the explicit discretization

$$
\begin{aligned}
\hat{u}_{k}=-10 \operatorname{sgn}\left(x_{2, k}+0.5 \operatorname{sgn}\left(x_{1, k}\right)\right) & \\
& -10 \cdot\left(x_{2, k}+0.5 \operatorname{sgn}\left(x_{1, k}\right)\right) .
\end{aligned}
$$

The results are shown in Figure 9. Equation (49) lacks a selection strategy based on the information available at time $t_{k}$, which explains the noticeable increase in chattering in the input and in the regulation variable.
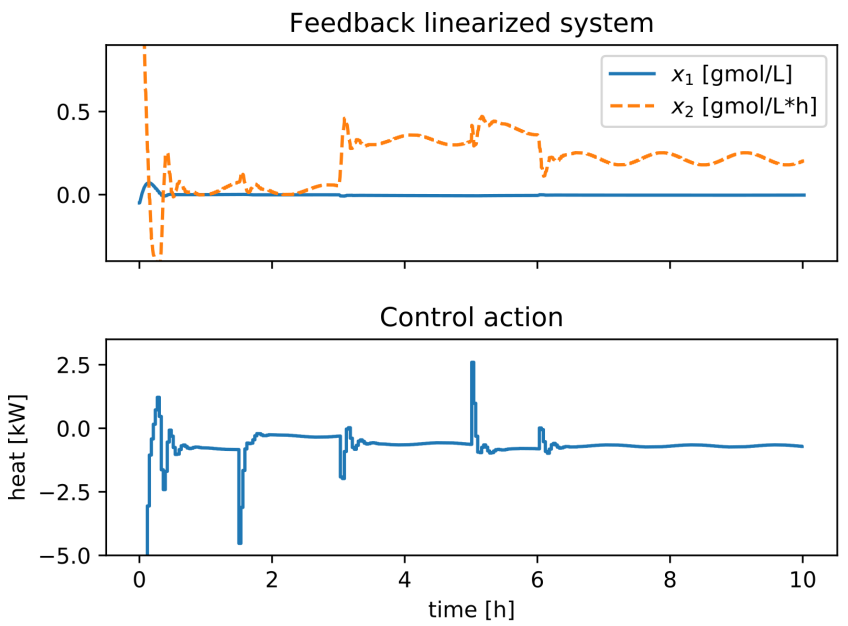

Fig. 8. Performance of (13) with implicit control selection (32), (36) and gains $\gamma_{1}=0.5, \gamma_{2}=10$ and $\gamma_{3}=10$. The controller keeps $x_{1}$ close to zero without significant chattering. The state $x_{2}$ compensates $\zeta_{1}$.
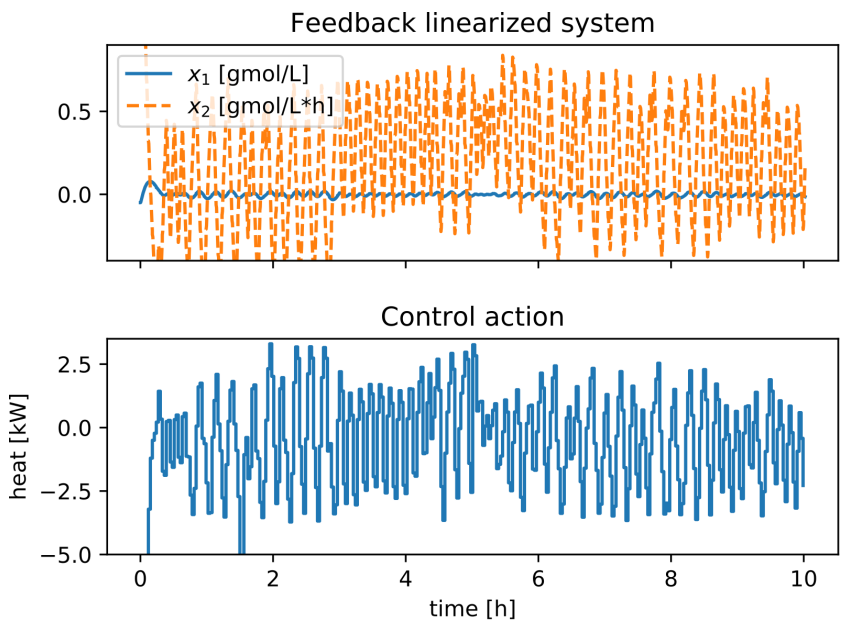

Fig. 9. Performance of (13) with explicit control (49). The controller keeps $x_{1}$ relatively close to zero, but at the expense of significant chattering in the control signal and in $x_{2}$.

\section{Discussion}

The key issue when discretizing the control law (14) is the interplay between controller gains, sampling time and regularization of the signum function, and this is where the main contribution of the paper resides. In the traditional approach, the controller in continuous time is first regularized and then discretized. The reader familiar with this approach could argue that the control law (49) can yield roughly the 'same level' of chattering as the implicit scheme by properly regularizing the signum function in continuous time. This argument is only partially true because, on one hand, an arbitrary regularization does not guarantee the reduction of chattering and, on the other hand, a correct tuning of the regularization (saturation or sigmoid or else) may be quite a difficult task, even in the simplest case of first-order 
SMC with a single sliding surface [20, Section 3.2.6], [4, Section II.B]. Namely, the smoothness of the control law depends on the required gains and the sampling period and, to the best of the authors knowledge, there is no systematic procedure for choosing the appropriate regularization based on these parameters, just trial and error by simulation. In the implicit method the regularization is not performed in continuous time, it is performed after the system has been discretized. As a result, the regularized control (32) with (36a) and (36b) is set with concrete parameters depending on the sampling period and the controller gains.

Finally, it is worth commenting on the plant's implicit/explicit discretization (29). Suppose that we fix the gains $\gamma_{i}$. Then, the model is consistent with (10) in the sense that, given the boundedness of the discrete solutions of (29), (32), (36), uniformly on $h$, it is not difficult to show that these converge to a solution of (16) as $h$ goes to zero. The proof follows along the lines of [21, Theorem 6]. Fixing the gains and taking the limit $h \rightarrow 0$ entails of course the loss of the discrete sliding motion but, as above, rejection of the unmatched disturbance is preserved without noticeable chattering.

\section{Conclusions and further work}

A set-valued nested controller was proposed. The controller ensures the robust regulation of the output in the presence of non-vanishing mismatched disturbances. The implemented controller uses an implicit discretization together with a backstepping-like algorithm for the selection of the control values. The proposed selection strategy exhibits a much better performance when compared with the explicit discretization.

Nonsmooth Lyapunov functions are proposed for continuous and discrete time. Set-valued analysis then provides a relation between the gains and the compact set to which the system trajectories ultimately converge. The gains can then be tuned in a simple but effective fashion.

A possible future direction is the study of integral nested controllers which would eliminate the reaching phase, i.e., the period of time taking place before the state enters $\mathcal{R}$. This would avoid the large initial peaks displayed by the control input. Also, the methodology could be extended to systems of relative degree higher than two.

\section{References}

[1] V. Utkin, J. Guldner, and J. Shi, Sliding Mode Control in Electro-Mechanical Systems. CRC press, 2009, vol. 34.

[2] J.-J. E. Slotine and S. S. Sastry, "Tracking control of non-linear systems using sliding surfaces, with application to robot manipulators," International Journal of Control, vol. 38, no. 2, pp. 465-492, 1983.
[3] A. Levant, "Introduction to high-order sliding modes," School of Mathematical Sciences, Israel, 2003.

[4] K. D. Young, V. Utkin, and Ü. Özgüner, "A control engineer's guide to sliding mode control," Control Systems Technology, IEEE Transactions on, vol. 7, pp. 328 - 342, Mar. 1999.

[5] F. Castaños and L. Fridman, "Analysis and design of integral sliding manifolds for systems with unmatched perturbations," Automatic Control, IEEE Transactions on, vol. 51, no. 5, pp. 853-858, 2006.

[6] J. Davila, "Exact tracking using backstepping control design and high-order sliding modes," Automatic Control, IEEE Transactions on, vol. 58, no. 8, pp. 2077-2081, 2013.

[7] A. Estrada and L. Fridman, "Quasi-continuous HOSM control for systems with unmatched perturbations," Automatica, vol. 46, no. 11, pp. 1916-1919, 2010.

[8] A. Ferreira, F. J. Bejarano, and L. Fridman, "Unmatched uncertainties compensation based on high-order sliding mode observation," International Journal of Robust and Nonlinear Control, vol. 23, no. 7, pp. 754-764, 2013.

[9] R. A. Freeman and P. Kokotović, Robust Nonlinear Control Design: State-space and Lyapunov Techniques, ser. Modern Birkhäuser Classics. Boston: Birkhäuser, 2008.

[10] T. Sadikhov and W. M. Haddad, "A universal feedback controller for discontinuous dynamical systems using nonsmooth control Lyapunov functions," Journal of Dynamic Systems, Measurement, and Control, vol. 137, no. 4, p. 041005, 2015.

[11] H. H. Choi, "An LMI-based switching surface design method for a class of mismatched uncertain systems," Automatic Control, IEEE Transactions on, vol. 48, pp. 1634-1638, Sep. 2003.

[12] F. A. Miranda-Villatoro, B. Brogliato, and F. Castaños, "Set-valued sliding-mode control of uncertain linear systems: continuous and discrete-time analysis," SIAM Journal on Control and Optimization, vol. 56, pp. 1756 - 1793, March 2018.

[13] S. T. Venkataraman and S. Gulati, "Control of nonlinear systems using terminal sliding modes," Journal of Dynamic Systems, Measurement, and Control, vol. 115, no. 3, pp. 554560, 1993.

[14] M. Zhihong, A. P. Paplinski, and H. R. Wu, "A robust MIMO terminal sliding mode control scheme for rigid robotic manipulators," Automatic Control, IEEE Transactions on, vol. 39, no. 12, pp. 2464-2469, 1994.

[15] V. Acary and B. Brogliato, "Implicit Euler numerical scheme and chattering-free implementation of sliding mode systems," Systems \& Control Letters, vol. 59, no. 5, pp. 284-293, 2010.

[16] R. Kikuuwe, S. Yasukouchi, H. Fujimoto, and M. Yamamoto, "Proxy-based sliding mode control: A safer extension of PID position control," Robotics, IEEE Transactions on, vol. 26, pp. $670-683$, August 2010.

[17] V. Acary, B. Brogliato, and Y. V. Orlov, "Chatteringfree digital sliding-mode control with state observer and disturbance rejection," Automatic Control, IEEE Transactions on, vol. 57, no. 5, pp. 1087-1101, 2012.

[18] S. Jin, R. Kikuuwe, and M. Yamamoto, "Improving velocity feedback for position control by using a discrete-time sliding mode filtering with adaptive windowing," Advanced Robotics, vol. 28, pp. 943 - 953, July 2014.

[19] O. Huber, V. Acary, and B. Brogliato, "Lyapunov stability and performance analysis of the implicit discrete sliding mode control," Automatic Control, IEEE Transactions on, vol. 61, no. 10, pp. 3016-3030, October 2016. 
[20] O. Huber, B. Brogliato, V. Acary, A. Boubakir, F. Plestan, and B. Wang, "Experimental results on implicit and explicit time-discretization of equivalent-control-based sliding mode control," in Recent Trends in Sliding Mode Control, L. Fridman, J.-P. Barbot, and F. Plestan, Eds. IET, 2016, pp. 207-236.

[21] F. A. Miranda-Villatoro, B. Brogliato, and F. Castaños, "Multivalued robust tracking control of Lagrange systems: Continuous and discrete-time algorithms," Automatic Control, IEEE Transactions on, vol. 62, pp. 4436 - 4450, September 2017.

[22] A. Adhami-Mirhosseini and M. J. Yazdanpanah, "Robust tracking of perturbed nonlinear systems by nested sliding mode control," in 2005 International Conference on Control and Automation, vol. 1, 2005, pp. 44-48.

[23] A. Levant, "Higher-order sliding modes, differentiation and output-feedback control," International Journal of Control, vol. 76, no. 9-10, pp. 924-941, 2003.

[24] N. Baiomy and R. Kikuuwe, "An amplitude- and ratesaturated controller for linear plants," Asian Journal of Control, vol. 21, pp. 1 - 15, Nov. 2019.

[25] A. Bacciotti and F. Ceragioli, "Stability and stabilization of discontinuous systems and nonsmooth Lyapunov functions," ESAIM: Control, Optimisation and Calculus of Variations, vol. 4, pp. 361-376, 1999.

[26] J. Aubin and A. Cellina, Differential Inclusions: SetValued Maps and Viability Theory, ser. Grundlehren der mathematischen Wissenschaften. Springer Berlin Heidelberg, 1984.

[27] V. A. Yakubovich, G. A. Leonov, and A. K. Gelig, Stability of Stationary Sets in Control Systems with Discontinuous Nonlinearities. Singapore: World Scientific, 2004.

[28] F. H. Clarke, Optimization and Nonsmooth Analisis. New York: Society for Industrial and Applied Mathematics, 1990.

[29] — Optimization and Nonsmooth Analysis, ser. Classics in Applied Mathematics. Philadelphia: SIAM, 1990.

[30] H. K. Khalil, Nonlinear Systems. Upper Saddle River, New Jersey: Prentice-Hall, 1996.

[31] A. Isidori, Nonlinear Control Systems. London, U.K.: Springer-Verlag, 1996.

[32] P. Daoutidis and C. Kravaris, "Synthesis of feedforward/state feedback controllers for nonlinear processes," AIChE Journal, vol. 35, pp. 1602 - 1616, Oct. 1989.

[33] G. V. Smirnov, Introduction to the Theory of Differential Inclusions. Providence, Rhode Island: American Mathematical Society, 2002.

[34] K. Deimling, Multivalued Differential equations. Berlin: Walter de Gruyter, 1992.

[35] H. Brézis, Operateurs Maximaux Monotones et Semi-Groupes de Contractions dans des Espaces de Hilbert. Amsterdam: North-Holland, 1973.

[36] J. V. Burke, M. C. Ferris, and M. Qian, "On the Clarke subdifferential of the distance function of a closed set," Journal of Mathematical Analysis and Applications, vol. 166, no. 1, pp. 199-213, 1992. 